\title{
DETC2008-50084
}

\section{INTERACTIVE DEFORMATION THROUGH MESH-FREE STRESS ANALYSIS IN VIRTUAL REALITY}

\author{
Daniela Faas \\ Department of Mechanical Engineering \\ Virtual Reality Applications Center \\ Iowa State University \\ Ames Iowa 50011 \\ dfaas@iastate.edu
}

\author{
Judy M. Vance \\ Program Director for Engineering Design \\ Civil, Mechanical, and Manufacturing Innovation Division \\ National Science Foundation \\ 4201 Wilson Blvd, Room 529 \\ Arlington, VA 22230 \\ jmvance@nsf.gov \\ ASME Fellow
}

\begin{abstract}
Virtual reality (VR) techniques are used to create an environment that allows an engineer to modify the shape of a part and see the changes in the stress state immediately. A virtual reality application, Interactive Virtual Design Application (IVDA), that allows fast mesh-free analysis of multiple element types, including two-dimensional (2D) elements, is described in detail. Taylor series approximations and Pre-conditioned Conjugate Gradient (PCG) methods are used with mesh-free analysis to perform quick reanalysis during interactive shape modification. Prior to this work, only 3D elements were incorporated into the method. The addition of 2D elements greatly expands the potential application of this work. Several software packages including VR Juggler, OpenHaptics, OPCODE, Tahoe and OpenGL/GLM/GLUT libraries are combined in the resulting application to handle a variety of elements. This approach also supports concurrent product design and assembly methods prototyping. The addition of 2D analysis capability is discussed in this paper. The method is described and a sample problem presented.
\end{abstract}

Keywords: Mesh-free analysis, Virtual Reality, Subdivision Volumes, Human Computer Interaction, Virtual Assembly, Mechanical Design.

\section{INTRODUCTION}

Virtual Reality, through the use of stereo viewing and position tracking, allows participants to use natural human motions to interact with computer models in a 3D space.
Although VR is used in the mechanical design process for prototyping, very few have combined it with free-form deformation and stress analysis. Combining virtual reality tools with finite element analysis allows for interactive, threedimensional visualization and exploration of potential design changes. When performed in a collaborative VR environment this presents the potential for bringing multiple members of the design team, including engineers, marketing experts, and manufacturing personnel, together to explore potential new designs. Replacing the 2D interface of the traditional computer monitor with large screen stereo display systems and other VR interfaces creates an environment where team members can interact with potential new designs in a natural way, promoting increased understanding of the geometry and implications of design changes. The ability to interactively change the geometry and see the impact on stresses produced in the design allows the team to explore many potential designs in a short period of time. The goal of this research is to create a design environment where design shape changes and stress approximations can occur simultaneously in an interactive manner.

The paper presents added features to IVDA. Previously, only 3D solid structures were able to undergo deformation in the application. The addition of 2D shells will increase the range of models that can be examined in IVDA. The goal of this research is to perform fast mesh-free stress analysis in a virtual environment. In addition, the test bed enables fast and intuitive free form shape deformation and shape evaluation through stress analysis and haptic feedback. 
The paper will describe the design environment and the underlying theory and algorithms.

\section{BACKGROUND}

Recently, virtual reality applications have become much more than fly-through or stereo viewing experiences. Complex data or geometry can be visualized, and $3 \mathrm{D}$ navigation, stereo immersive viewing and position tracking allow the VR environment to be used as an interactive, intuitive design tool. In particular, the Cave Automatic Virtual Environment (CAVE ${ }^{\mathrm{TM}}$ ) allows the user to stand in a full-size room with up to 6 screens. The C6 at Iowa State's Virtual

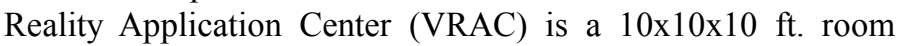
where stereo images are projected on all four walls, the ceiling, and the floor [1] (Figure 1). The six projection surfaces each display 4096x4096 pixels, making it the highest resolution CAVE system in the world with over 100 million pixels of total resolution.



Figure 1: The C6 in the Virtual Reality Applications Center.

Virtual Reality (VR) has been used in the mechanical design process for prototype evaluation and assembly methods prototyping [2-6]. Dai and Goebel first suggested the use of the virtual reality environment to visualize finite element results in 1994 [5]. Finite element visualization was researched by Plaskacz et al. in 1996 [7] and others. These applications focused on simply displaying the results of an analysis, not interacting with the model itself.

In 1998 Yeh and Vance [8] were the first to combine virtual reality with free form deformation to perform interactive stress analysis in virtual reality. Linear Taylor series approximations were used to calculate the new stress values during interactive shape deformation based on pre-computed stress-sensitivities. A rectangular Non-Uniform Rational BSpline (NURBS) [9] bounding volume defined the region in which the deformation could occur (Figure 2 and 3). Ryken and Vance then used this method in an industrial application to redesign the lift arm of a John Deere tractor [10].

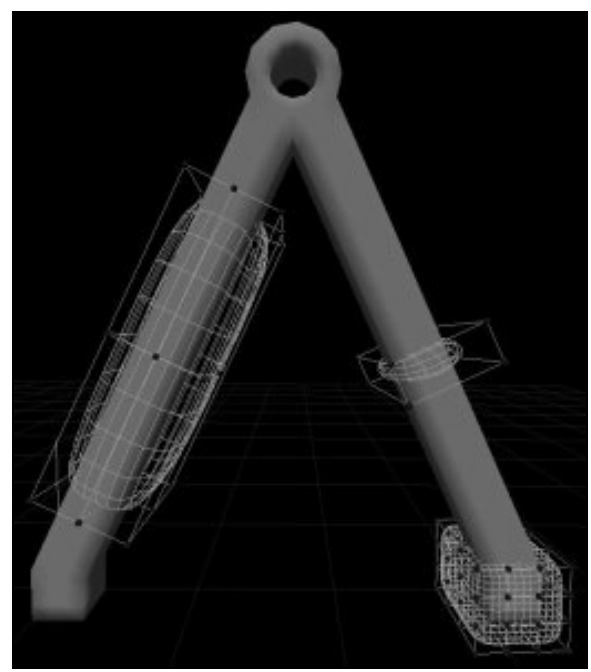

Figure 2: Subdivided bounding volumes around a model in the virtual environment.



Figure 3: User selects bounding volume control points.

Further research resulted in methods that allowed more accurate stress approximations for large design changes. Chipperfield et al. [11] implemented a mesh-free solver that was embedded into the application. Mesh-free approximation provides the capability to perform large design changes without mesh distortion affecting the analysis results. Automated remeshing is not a viable option for this test bed. Because virtual reality requires real-time computation. Re-meshing can be very compute intensive and therefore, this VR testbed required an analysis method which could handle large deformations without automated re-meshing. A time comparison between traditional FEA and the mesh-free method was explained in [13].

The reproducing kernel particle method (RKPM) with strain smoothing stabilization, introduced by Chen et al [18] was selected as the mesh-free approach for this work. The RKPM is used to approximate unknown displacements in terms of the displacement coefficients at the mesh-free nodes. The displacement is defined as: 


$$
u^{h}(\mathbf{x})=\sum_{I=1}^{N} \Psi_{I}(\mathbf{x}) d_{I}
$$

where $u^{h}(x)$ is the displacement, $\Psi_{I}(x)$ is the reproducing kernel shape function evaluated at the point x, with respect to the $I^{\text {th }}$ node, and $d_{I}$ are the displacement coefficients. Furthermore, the strain is defined as:

$$
\boldsymbol{\varepsilon}^{h}\left(\mathbf{x}_{L}\right)=\sum_{I \in G_{L}} \mathbf{B}_{I}\left(\mathbf{x}_{L}\right) \mathbf{d}_{I}
$$

where $\boldsymbol{\varepsilon}^{h}\left(\mathrm{x}_{\mathrm{L}}\right)$ is the strain at node $L, \mathrm{~B}_{I}$, the smoothed strain gradient matrix; and $d_{I}$, the vector of displacement coefficients for node $I$. The function $u^{h}(\mathrm{x})$ is approximated using the surrounding particles using RKPM shape functions. The meshfree method has been shown to be accurate in numerous books and journals $[19,20]$ and for mesh-free analyses of shells as well: [21-24]. Mesh-free solvers have been evaluated and tested for this particular application [26].

In addition, a PCG reanalysis method was added to rapidly and accurately compute the stress contours resulting from larger design changes without recalculating the entire matrix of equations [13]. Chipperfield et al. developed a two step process to approximate the stresses. The Taylor series approximation, was used as the designer interactively changed the shape of the geometry. When a suitable shape was achieved, the PCG method calculated more accurate stress values. The Taylor series approximation allows for quick, real-time stress updates as the user changes the shape in the virtual environment, while the PCG method allows for a more accurate reanalysis after the interactive changing is completed.

The Conjugate Gradient (CG) Method is an iterative method for solving sparse systems of linear equations. CG is effective for systems of the form

$$
\mathbf{A} x=b \quad \text { Eq. } 3
$$

Where $x$ is an unknown vector, $b$ is a known vector, and $\mathbf{A}$ is a known, square, symmetric, positive-definite matrix.

Conjugate gradient is a robust technique, but for large problems becomes a slow process. Therefore, preconditioning

\begin{tabular}{|c|c|c|}
\hline \multirow{4}{*}{$\begin{array}{l}\text { Where } \\
\text { And }\end{array}$} & $\mathbf{A}^{\prime} x^{\prime}=b^{\prime}$ & Eq. 4 \\
\hline & $\left(\mathbf{Q}^{-1} \mathbf{A} \mathbf{Q}^{-\mathbf{T}}\right)\left(\mathbf{Q}^{\mathbf{T}} x\right)=\mathbf{Q}^{-1} b$ & Eq. \\
\hline & $\mathbf{A}^{\prime}=\mathbf{Q}^{-1} \mathbf{A} \mathbf{Q}^{-\mathbf{T}}$ & Eq. 6 \\
\hline & $\begin{array}{l}x^{\prime}=\mathbf{Q}^{1} \\
b^{\prime}=\mathbf{Q}^{-1} b\end{array}$ & $\begin{array}{l}\text { Eq. } 7 \\
\text { Eq. } 8\end{array}$ \\
\hline
\end{tabular}
the system lowers the order and reduces the solution time which results in rapid convergence. Preconditioning allows the explicit solution of:
The matrix $\mathbf{Q}$ is to be chosen to be nonsingular matrix [14-17]. In this application, the previous stiffness matrix was used as the preconditioning matrix for the next set of calculations.

\section{INTERACTIVE VIRTUAL DESIGN APPLICATION}

IVDA was developed using the VR Juggler open source software toolkit (www.vrjuggler.org) and provides an application interface that supports a wide variety of display devices $[27,28]$. The application can run on a desktop monitor, one wall projection screen, multiple wall projection screens or a head mounted display by selecting different configuration files.

Designers exploring shape changes must also consider the effect of these geometry changes on other parts in an assembly. The OPCODE (Optimized Collision Detection) library [29] has been incorporated into IVDA so that several models can be analyzed and assembled without models intersecting with each other $[30,31]$. This software identifies collisions between deformable meshes and therefore is well suited for this work.

IVDA also has the ability to incorporate haptic feedback into the design experience. Haptic devices are used in a wide variety of virtual reality applications [32-39]. In IVDA, in addition to using a position-tracked wand, the engineer can also select to use the SensAble Technology PHANTOM ${ }^{\mathrm{TM}}$ Haptic device [40] to modify the model, select different control points, change bounding volumes, and explore the shape change effects on the part in question [30] (Figure 4).

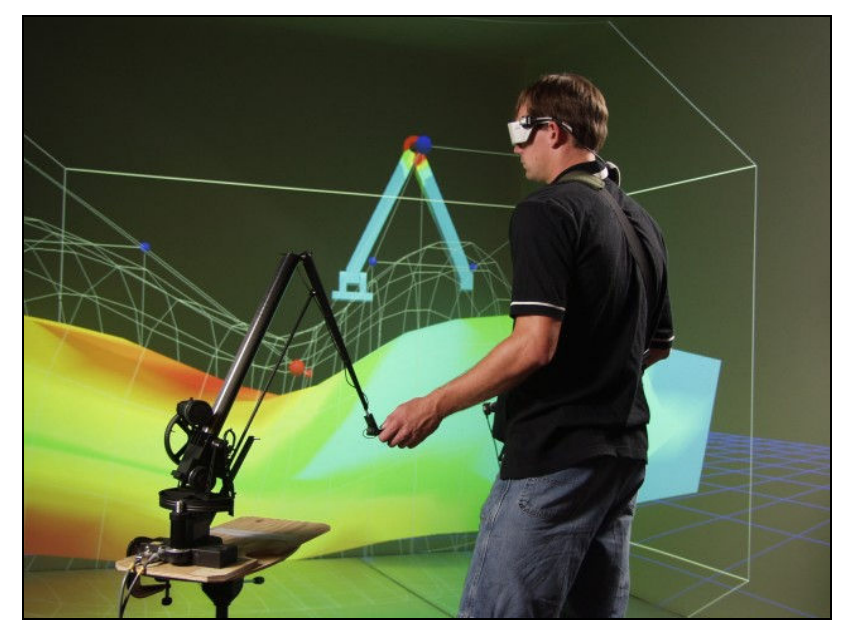

Figure 4: A user working with IVDA and the PHANTOM haptic device.

The haptic device is used as a simple 3D cursor input but also to provide force feedback to the user. The force generated is proportional to the level of stress in the elements in the area of the haptic cursor. Higher stresses are modeled with a stiffer spring force. The haptic device resists model deformation when the stresses become larger as the user deforms the model. Because higher stresses result in higher force feedback, the engineer will gain a more intuitive understanding of the level of stress induced in the model as a 
result of shape changes. Because of the need for $1000 \mathrm{~Hz}$ haptic rates, a separate computer was used to drive the PHANTOM and networked with the cluster driving the application [35].

An open source software package, Tahoe, was selected as the mesh-free solver. Tahoe supports many different elements, material models, and analysis types, including meshfree elements, crack analysis, cohesive models and a number of other more specialized analysis models [42]. The geometry and material file formats are created in ABAQUS [43] and converted to Tahoe input format in a pre-processing procedure . These input files contain the geometry (nodal positions), a mesh-free nodal arrangement for integration points, material, and boundary and load conditions.

Figure 5 presents a flowchart outlining the IVDA process. Once the model is loaded into IVDA, an initial stress analysis is performed. The user then is able to view the model with the contoured Von Mises stresses. A bounding volume can be defined around an interest region of the part where a deformation is desired. The bounding volume is created using Catmull-Clark subdivision methods. The user selects two points in space using the wand or the haptic device, which define the extents of the volume. The user can then choose to subdivide the volume to achieve smoother shape deformations. Multiple volumes can be created on a single model in the event that there are several areas of interest. The model can then be deformed. As the geometry changes, the stresses are updated simultaneously using a Taylor series approximation. Once the engineer is satisfied with the geometric changes, the model is reanalyzed using the PCG method.

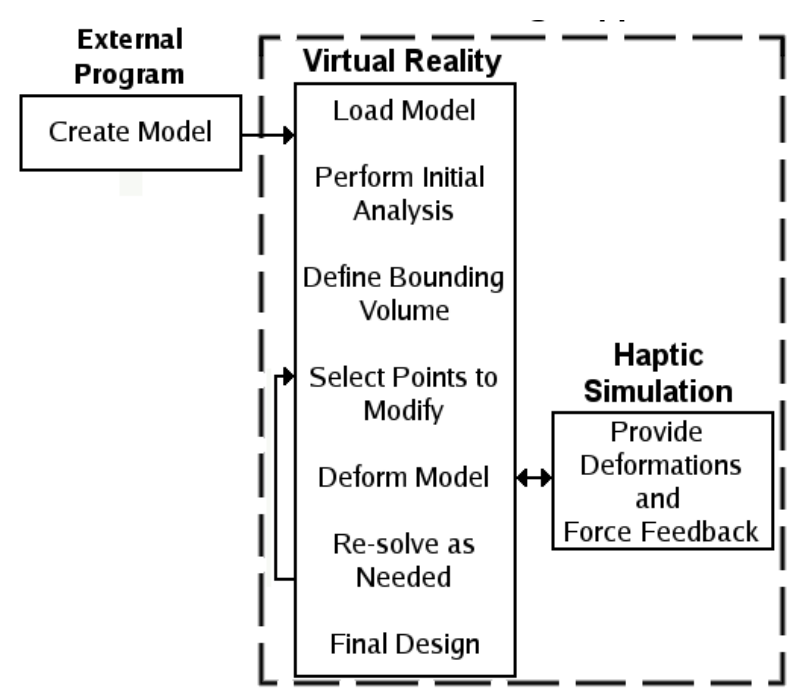

Figure 5: Flowchart for IVDA process.

An output option allows the engineer to export the new nodal coordinates, which are then reassembled into the corresponding element for analysis with ABAQUS. There is no significant difference between the analyses performed in IVDA or in ABAQUS. Large deformation of the nodes in IVDA can potentially lead to badly meshed elements in ABAQUS when exported. An element check for angle and aspect ratio should always be performed when using the exported nodal coordinates from IVDA to reduce instabilities due to bad elements.

\section{D EXAMPLE}

The current work has extended the available elements in IVDA to include 1D and 2D elements. Previous versions of IVDA were not able to display and perform a mesh-free analysis on other elements besides 3D. The 2D elements are read into IVDA and the model is flagged as a 2D model. The information sent to Tahoe is $2 \mathrm{D}$, while the model display in VR is in a 3D format. During the PCG analysis, the data send to Tahoe is flagged as 2D or 3D based on the initial flag. Table 1 lists the elements which are supported in IVDA.

Table 1: Element Models

\begin{tabular}{|c|c|c|}
\hline Geometry & Dimensions & \# of Nodes \\
\hline point & 1 & 1 \\
line & 1 & 2 \\
quadrilateral & 2 & 4,8 \\
triangle & 2 & 3 \\
hexahedron & 3 & 8 \\
tetrahedron & 3 & 4 \\
pentahedron & 3 & 6 \\
\hline
\end{tabular}

An engineer wants to explore the design of a stepped plate. The preliminary finite element analysis shows that the stresses are too high, but the engineer is not sure how to reduce the stresses in the part. IVDA allows the engineer to get a "feel" for stresses by allowing shape deformation and simultaneously computing the stresses. Prior to using IVDA, there is no connection between the stress and the shape. Once the stresses are reasonable and the shape looks good to the engineer, the new model can be exported from IVDA into a typical finite element analysis program such as ABAQUS.

The 2D plate model as shown in Figure 6 is used as an example. The model consists of a plate of constant thickness, $\mathrm{h}=1 \mathrm{~mm}$, and length $\mathrm{L}=400 \mathrm{~mm}$, with two areas of different width, $\mathrm{w} 1=100 \mathrm{~mm}$ and $\mathrm{w} 2=50 \mathrm{~mm}$. The plate is modeled with 2D triangular "elements" and is fixed on one end and a constant, fixed displacement of $100 \mathrm{~mm}$ is applied to the other end.

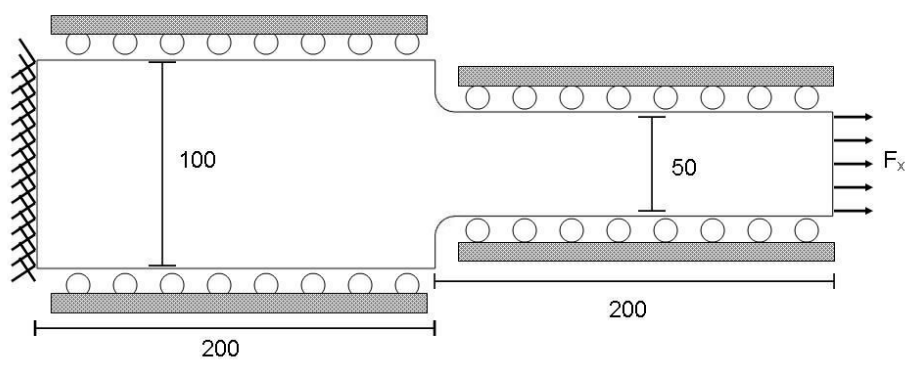

Figure 6: Example problem. 
The plate is well within the scope of Kirchhoff's theory. The Young's modulus is $\mathrm{E}=10^{8} \mathrm{~Pa}$ and the Poisson's ratio is $\mathrm{v}=0.35$. Figure 7 shows the initial finite element analysis in ABAQUS. Previous papers have discussed the accuracy of the mesh-free method. A paired t-test was performed with a significance level of 0.5 and no significant difference was found between the analyses performed in IVDA or in ABAQUS. The analysis performed in IVDA performs highly accurate and no significant difference was noted.



Figure 7: Von Mises Stress Results in ABAQUS.

The first step is to create the bounding volume. The Bounding Volume sub-menu appears and "Create New Bounding Volume" is selected. The user points the wand on the part. The user completes the bounding volume when the wand is used as a pointer to select two positions in space that define the extents of the bounding volume. More points can be added to the bounding volume, making further refinements to the subdivision volume (Figure 8).

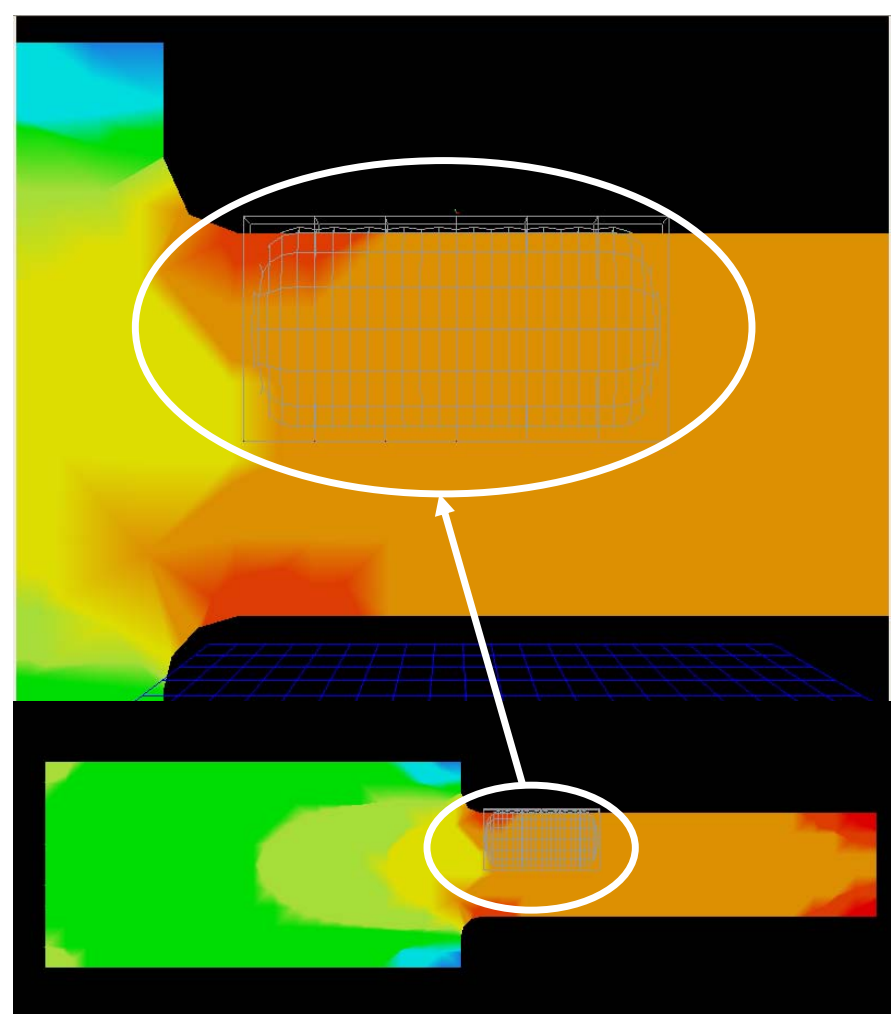

Figure 8: Bounding Volume with several subdivisions in IVDA.

The user can deform the model by selecting the desired control points with the wand. Using natural hand-movements, the control points are moved in a truly three dimensional environment (Figure 9 and 10). The user can deform the model in 6 degrees of freedom.



Figure 9: Localized design changes are based on bounding volume. 


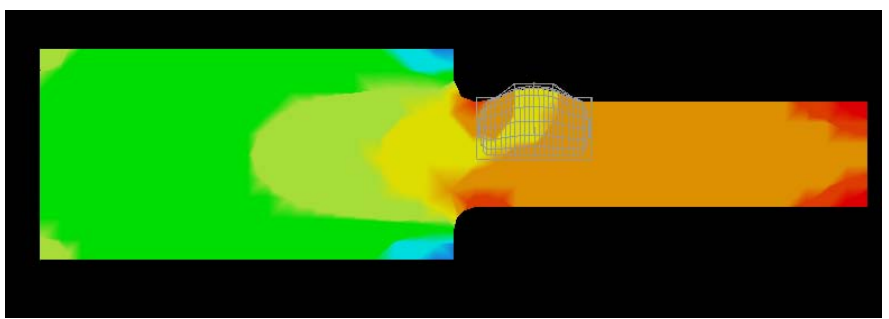

Figure 10: Final Design in IVDA.

During the interactive exploration, the stresses are updated using a Taylor series approximation. Once a suitable geometry is achieved, the PCG method is used to solve the systems of equations for more accurate stresses. The user is able to deform the part until a desired shape is achieved. Since the stresses are updated interactively, the engineer can explore multiple shape changes and gain an understanding of the effect these changes have on the stresses produced in the part.

\section{CONCLUSION}

In sum, IVDA allows engineers to work together on fast interactive investigations of multiple part shapes early in the product design process. More design options can be explored with IVDA when analysis results are accurately displayed in real-time. In particular, the mesh-free method supports large deformations. The use of the open-source software Tahoe allows for the stress computation of solid and shell elements using mesh-free techniques. The addition of the $2 \mathrm{D}$ elements opens up the application to allowing for a wide variety of design models to be examined.

The force feedback through the PHANTOM haptic device gives the designer even more information on the affect of design changes on the stress situation of the part. Because of the intuitive interaction provided by the immersive virtual reality application, this implementation encourages creativity and the opportunity for many design options to be explored.

The combination of immersive design team participation, intuition, experience and domain-specific knowledge will allow members of the design team to more fully understand the implications of design changes early in the design process. This has the potential to greatly impact product design and result in the design of products that require less redesign later in the design process.

\section{FUTURE WORK}

Future work will involve refinement of the bounding volume creation and manipulation as well as improvements to the interactive analysis and haptic feedback force modeling during assembly operations.

\section{ACKNOWLEDGMENTS}

The authors would like to thank the Iowa State University's Virtual Reality Applications Center for the use of computational resources and hardware. This research was funded by The Procter \& Gamble Company.

\section{DISCLOSURE}

Any opinion, findings, conclusions, and recommendations expressed in this material are those of the authors and do not necessarily reflect the views of the National Science Foundation.

\section{REFERENCES}

1. Center, Virtual Reality Applications, C6, Iowa State University, 2008, http://www.vrac.iastate.edu/c6.php.

2. Gupta, Rakesh, Thomas Sheridan, and Daniel Whitney, Experiments Using Multimodal Virtual Environments in Design for Assembly Analysis. Presence, 1997. 6(3): p. 318-338.

3. Jayaram, Sankar, Hugh I. Connacher, and Kevin W. Lyons, Virtual assembly using virtual reality techniques. Computer-Aided Design, 1997. 29(8): p. 575-584.

4. Jayaram, Sankar, Judy Vance, Rajit Gadh, Uma Jayaram, and Hari Srinivasan, Assessment of VR Technology and its Applications to Engineering Problems. Journal of Computing and Information Science in Engineering, 2001. 1: p. 72-83.

5. Dai, Fan and Martin Goebel. Virtual Prototyping - an Ap-proach using VR-techniques. in Proceedings of the 14th ASME International Computers in Engineering Conference. 1994. Minneapolis, Minnesota.

6. Antonya, Csaba and Doru Talaba, Design Evaluation and modification of mechanical systems in virtual environments. Virtual Reality, 2007. 11(4): p. 275-285.

7. Plaskacz, Edward J. and Roland F. Kulak. Use of highperformance computers, FEA and the CAVE automatic virtual environment for collaborative design of complex systems. in ASME design engineering show and conference, Chicago, IL (United States). 1996.

8. Yeh, Tsun-Pin and Judy M. Vance, Applying Vitrual Reality Techniques to Sensitivity-Based Structure Shape Design. ASME Journal of Mechanical Design, 1998.120 (4): p. 612-619.

9. Piegl, Les and Wayne Tiller, The NURBS Book. Vol. 2. 1997: Springer-Verlag Berlin Heidelberg.

10. Ryken, Michael J. and Judy M. Vance, Applying Virtual Reality Techniques to the Interactive Stress Analysis of a Tractor Lift Arm. Finite Elements in Analysis and Design, 2000. 35: p. 141-155.

11. Chipperfield, Kurt and J. Vance. Virtual Reality Interactive Design Utilizing Meshless Stress ReAnalysis. in ASME 2005 International Design Engineering Technical Conferences \& Computers and Information in Engineering Conference, September 24-28. 2005. Long Beach, California, DETC200584496 
13. Chipperfield, Kurt, Judy M. Vance, and Andrew Fischer, Fast Meshless Reanalysis Using Combined Approximations, Preconditioned Conjugate Gradient, and Taylor Series. AIAA Journal, 2006. 44(6): p. 1325-1331.

14. Atkinson, Kendall, Introduction to Numerical Analysis. 1989.

15. Golub, Gene, Matrix Computations. 1996, Baltimore, MD: Johns Hopkins University.

16. Hackbusch, Wolfgang, Iterative Solution of Large Space Systems of Equations. 1994, New York,NY: Springer-Verlag.

17. Avriel, Mordecai, Nonlinear Programming, Analysis and Methods. 2003, Mineola, NY: Dover Publications, Inc.

18. Chen, Jiun-Shyan, Cheng-Tang Wux, Sangpil Yoon, and Yang Youk, A stabilized conforming nodal integration for Galerkin mesh-free methods. International Journal for Numerical Methods in Engineering, 2001(50): p. 435-466.

19. Shapiro, Vadim and Igor Tsukanov, Meshfree Simulation of Deforming Domains. CAD Computer Aided Design, 1999(31:77): p. 459-471.

20. Liu, G. R., Adaptive topology optimization of elastoplastic structures. 2003, Danvers, MA: CRC.

21. Guo, X., Xin Li, Yunfan Bao, X. Gu, and Hong Quin, Meshless Thin-Shell Simulation based on Global Conformal Parameterization. IEEE Transactions on Visualization and Computer Graphics, 2006. 12(3): p. 375-385.

22. Noguchi, H., T. Kawashima, and T. Miyamura, Element Free Analysis of shell and spatial structures. International Journal for numerical methods in Engineering, 2000. 47: p. 1215-1240.

23. Wang, Dongdong and Jiun-Shyan Chen, Locking-free stabilized conforming nodal integration for meshfee Mindlin-Reissner plate formualation. Comput. Methods Appl. Mech. Engrg., 2004. 193: p. 1065-083.

24. Kim, Nam Ho, Kyung Kook Choi, Jiun-Shyan Chen, and Mark E. Botkin, Meshfree analysis and design sensitivity analysis for shell structures. International Journal for numerical methods in Engineering, 2002. 53: p. 2087-2116.

26. Fisher, A. and J.M. Vance, Stress Sensitivity Calculation Methods for Interactive Mesh-Free Reanalysis. Computer Modeling and Simulation Engineering, 2007.

27. Bierbaum, Allen and Carolina Cruz-Neira. Runtime Reconfiguration in VR Juggler. in 4th Immersive Projection Technology Workshop. 2000. Ames, IA.

28. Bierbaum, Allen, Christopher Just, Patrick Hartling, Kevin Meinert, Albert Baker, and Carolina Cruz-Neira. VR Juggler: A Virtual Platform for Virtual Reality Application Development. in Virtual Reality. 2001. Yokohama, Japan.
29. Terdiman, Pierre, OPCODE User Manual. 2002.

30. Fischer, Andrew and Judy M. Vance, PHANTOM Haptic Device Implemented in a Projection Screen Virtual Environment, in 7th International Immersive Projection Technologies Workshop. 2003: Zurich, Switzerland.

31. Faas, Daniela, Andrew Fischer, and Judy M. Vance. Interactive mesh-free stress analysis for mechanical design assembly with Haptics. in ASME 2007 International Design Engineering Technical Conferences \& Computers and Information in Engineering Conference. 2007. Las Vegas, NV, DETC-DAC-34660.

32. Burdea, Grigore C. Haptics Issues in Virtual Environments. in CGI 2000. 2000. Geneva, Switzerland: IEEE.

33. Chipperfield, Kurt, Tsun-Pin Yeh, and Judy M. Vance. Interactive product development in a virtual environment using Haptics. in NSF Design, Service and Manufacturing Grantess and Research Conference. 2002. San Juan, Puerto Rico.

34. Harding, C. and M.C. Newcomb. Supporting Interactive Data Exploration for GIS Planning Tasks with a Multi-modal Virtual Environment. in Third IEEE Workshop on Haptic, Audio and Visual Environments (HAVE). 2004.

35. Kim, ChangEun and Judy M. Vance. Development of a networked haptic environment in VR to facilitate collaborative design using Voxmap Pointshell (VPS) software. in IMECE2005 ASME International Mechanical Engineering Congress and Exposition. 2005. Orlando, Florida, DETC-CIE-57648.

36. Massie, Thomas and J.K. Salisbury. The PHANTOM haptic Interface: A Device for Probing Virtual Objects. in ASME Symposium on Haptic Interfaces for Virtual Environments. 1994. Chicago, IL: ASME.

37. Salisbury, J. Kenneth and Mandayam A. Srinivasan, Phantom-Based Haptic Interaction with Virtual Objects. IEEE Computer Graphics and Applications, 1997. 17(5): p. 6-10.

38. Volkov, Sergei A. and Judy M. Vance, Effectiveness of Haptic Sensation for the Evaluation of Virtual Prototypes. Journal of Computing and Information Science in Engineering, 2001. 1(2): p. 123-128.

39. Bordegoni, M. and U. Cugini, Haptic modeling in the conceptual phases of product design. Virtual Reality, 2006. 40(2): p. 192-202.

40. Sensable, FreeForm Systems. 2006.

42. Sandia, National Laboratories, Tahoe Users guide. 2003.

43. Abaqus, http://www.simulia.com/, 2007. 